Dialectologia 25 (2020), 87-113.

ISSN: 2013-2247

Received 19 February 2019.

Accepted 20 May 2019.

\title{
À PROPOS DES NOMS DU RÂTEAU ET DE LA HERSE EN OCCITAN ARANAIS. ÉTUDE DE MICRODIALECTOLOGIE GASCONNE
}

\author{
Aitor CARRERA \\ Universitat de Lleida * \\ aitor.carrera@filcat.udl.cat
}

\section{Résumé}

Cet article porte sur les désignations de deux outils agricoles en gascon aranais : le râteau et la herse. Comme prévu, pour connaître les dénominations de ce genre d'instruments ayant trait à la vie traditionnelle, on doit s'en remettre aux recherches de Joan Coromines sur l'aranais. Mais, dans ce cas, on y décèle des manques, des lacunes très importantes, parfois des contradictions. Après avoir réalisé de nombreuses enquêtes dialectologiques dans le Val d'Aran, on est en mesure de dresser le répertoire des mots désignant ces outils et de préciser leur répartition géographique, qui s'avère assez complexe. Au total, on compte une demi-douzaine de dénominations, dont certaines ne figurent même pas chez Coromines. On va profiter de l'occasion pour en apprendre plus sur la position linguistique des parlers aranais et sur les rapports qu'ils entretiennent avec les dialectes gascons situés de l'autre côté de la frontière politique.

Mots clé

occitan, gascon, aranais, dialectologie, lexique

* Càtedra d'Estudis Occitans, Departament de Filologia Catalana i Comunicació, Universitat de Lleida. Plaça Víctor Siurana, 1. 25003 Lleida (Catalunya) 


\title{
THE DESIGNATIONS OF THE RAKE AND THE HARROW IN ARANESE OCCITAN. A GASCON MICRODIALECTOLOGY STUDY
}

\begin{abstract}
This article deals with two designations of agricultural instruments in Aranese Gascon: the rake and the harrow. As expected, to know the names of this type of instruments related to traditional life, we must begin with with Joan Coromines' research on Aranese. But in this case, we check insufficient data as well as very important gaps, even contradictions. After having carried out numerous dialectological surveys in the Val d'Aran, we can establish the inventory of forms that refer to these utensils and to specify their geographical distribution, which is certainly complex. We count up to a half dozen denominations, some of which do not even appear in Coromines's work. We are able to make some considerations on the linguistic position of the Aranese languages and on the relations they maintain with the Gascon dialects located on the other side of the political border.
\end{abstract}

\section{Keywords}

Occitan, Gascon, Aranese, dialectology, lexicon

\section{LAS DESIGNACIONES DEL RASTRILLO Y DE LA GRADA EN OCCITANO ARANÉS. ESTUDIO DE MICRODIALECTOLOGÍA GASCONA}

\section{Resumen}

Este artículo se ocupa de dos designaciones de instrumentos agrícolas en gascón aranés: el rastrillo y la grada. Como era previsible, para conocer las denominaciones de este tipo de instrumentos relacionados con la vida tradicional, debemos atenernos, para empezar, a las investigaciones de Joan Coromines sobre el aranés. Pero en este caso observamos una insuficiencia de datos así como lagunas muy importantes, incluso contradicciones. Después de haber realizado numerosas encuestas dialectológicas en el Valle de Arán, estamos en condiciones de establecer el inventario de formas que se refieren a dichos utensilios y de precisar su distribución geográfica, que se muestra ciertamente compleja. En total, contabilizamos hasta una media docena de denominaciones, algunas de las cuales ni tan siquiera figuran en la obra de Coromines. Va a ser también la ocasión para hacer algunas consideraciones sobre la posición lingüística de las hablas aranesas y sobre las relaciones que mantienen con los dialectos gascones situados al otro lado de la frontera política.

\section{Palabras clave}

occitano, gascón, aranés, dialectología, léxico 


\section{Présentation}

Les noms des outils agricoles sont un sujet récurrent dans les atlas linguistiques. En effet, en ce qui concerne le gascon, bon nombre de cartes de l'Atlas linguistique de la Gascogne portent sur cette question. Mais, pour ce qui est du Val d'Aran, étant donné que le seul atlas linguistique s'intéressant spécifiquement à l'aranais (l'Atlas lingüístic de la Vall d'Aran de Griera 1973 ; ALVA) contient de nombreuses erreurs et qu'il ne semble pas trop se pencher sur ce point, la source la plus fiable pour connaître ce genre de dénominations reste la monographie aranaise de Joan Coromines (1990), notamment son dictionnaire (Coromines 1990 : 259-756). On pourrait également évoquer une ribambelle de publications qui nous fournissent des données plus ou moins irrégulières sur le lexique du gascon parlé en Catalogne (et, du coup, sur la dialectologie aranaise), mais aucune de ces productions ne réussit à égaler celle de Coromines. Pourtant, en matière de désignations du râteau et de la herse en aranais, les informations de ce savant catalan nous paraissent tantôt lacunaires, tantôt contradictoires. Après vingt ans d'enquêtes dans le Val d'Aran, qui ont été réalisées dans dix-sept villages de ce pays gascon ${ }^{1}$ (dans le but d'en publier, un jour, un nouveau atlas linguistique), on est en mesure d'élargir de manière substantielle ce qui a été dit jusqu'ici sur les noms de ces deux instruments. Selon l'état actuel de nos recherches, l'occitan du Val d'Aran connaît jusqu'à une demidouzaine de termes désignant soit le râteau - notamment le râteau traditionnel en bois, utilisé pour le foin ou pour la paille, généralement muni de nombreuses dents -, soit la herse (dont la moitié n'ont pas été enregistrés par Coromines, du moins dans ces acceptions) : (ar)rastèth, pletera, (ar)rascle, carràs, èrsa et arpèga. Nul doute que la répartition géographique de ces mots - qui s'avère assez complexe -, ainsi que leur

\footnotetext{
${ }^{1}$ Tous les terçons (les anciennes subdivisions administratives du Val d'Aran) y ont été représentés : Es Quate Lòcs (Canejan, Bausen, Les, Bossòst) ; Lairissa (Arres, Vilamòs, Es Bòrdes) ; Marcatosa (Aubèrt) ; Castièro (Vielha, Betren, Escunhau) ; Arties e Garòs (Arties, Garòs) ; Pujòlo (Gessa, Salardú, Tredòs, Bagergue). Des enquêtes ont été également réalisées en Bavarthès (canton de Saint-Béat, département de la HauteGaronne). Pour plus de détails, voir Carrera (2017). Pour le terçon de Marcatosa (le berceau du poète aranais Jusèp Condò), on avait également prévu des enquêtes dans le village de Vilac (plus oriental qu'Aubèrt), mais on a du renoncer à un tel objectif compte tenu du manque d'informateurs remplissant toutes les conditions requises au moment d'achever nos interviews. Du coup, Aubèrt est devenu la localitétémoin de ce terçon-là.
} 
signifié (l'un d'entre eux, (ar)rascle, pouvant faire référence aux deux utensiles, mais pas au même endroit), vont nous aider à mieux comprendre la position des parlers aranais dans le contexte gascon et même dans l'ensemble de la langue occitane.

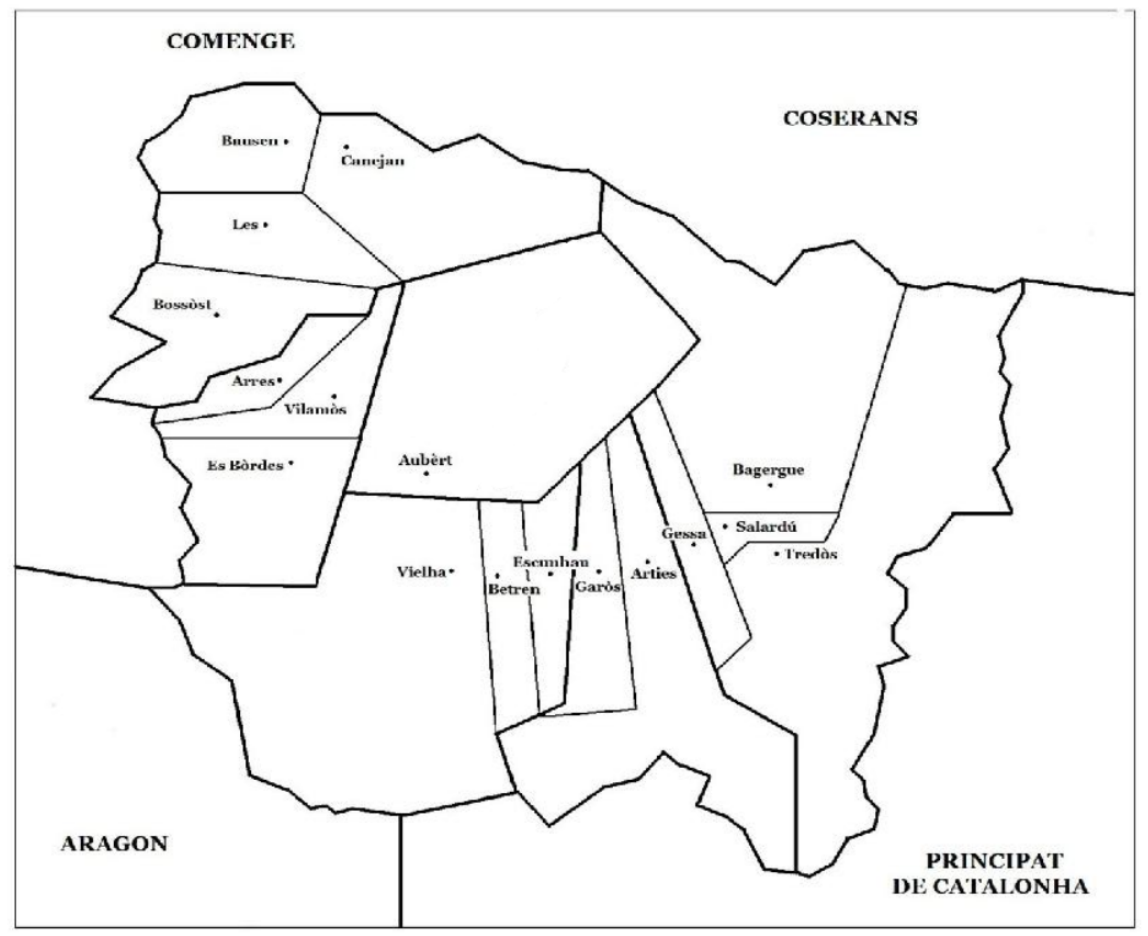

Carte 1. Points d'enquête

\section{2. (Ar)rastèth, 'râteau'}

Dans son dictionnaire aranais, Joan Coromines (1990: 285) consacre une entrée à (ar)rastèth :

RASTÈT [sic] m. Puj [Pujòlo], CV [centre de la vallée], arrastètx Cnj [Canejan], SJT [Sant Joan de Toran], 'eina en forma d'aixada, amb (dues o) tres pues de ferro, en lloc de la pala, que serveix per recollir fems'. A SJT 'rampí de recollir herba' ; i eğ 
arastę́d dera 'skía 'l'espinada' (literalment 'el rastell de l'esquena'). ${ }^{2}$ ETIM. = cat. rastell

D'après ce savant catalan, le terme (ar)rastèth - dont le référent n'aurait que deux ou trois dents - serait en usage un peu partout dans le Val d'Aran, ${ }^{3}$ mais lui-même, à propos d'un autre mot désignant le râteau, pletera (que nous allons examiner plus bas, $\S$ 3), restreint arrastèth aux villages du bassin du Toran (ruisseau qui se jette dans la Garonne à Pontaut), c'est-à-dire à Canejan et à Sant Joan de Toran : « A la mateixa pregunta contestaren [...] a Cnj i SJT arastẹč » (Coromines 1990 : 647). Ce mot servirait à nommer - sans allusion au nombre de dents - un « rascle amb pues de fusta o ferro, per arreplegar l'herba o esbarriar la terra ».

Dans sa thèse de doctorat, Coromines (1931 : 11) attribuait déjà arrastèth [aras'tet/] à Sant Joan de Toran, " rastro para recoger la hierba y otros usos ». D'ailleurs, il aurait entendu [ras'tet] à Bossòst (village disparu de la monographie de 1990), à Montcorbau (dans le centre du Val d'Aran) et à Tredòs (dans la haute vallée). Dans ces trois villages, cependant, ce mot indiquerait encore une fois une " herramienta a modo de azada, con tres púas de hierro en vez de pala ; se emplea para recoger estiércol ». L'attribution du terme à Montcorbau pourrait découler - comme à l'accoutumée - du vocabulaire du poète Jusèp Condò (1914 : 23), ${ }^{4}$ qui de son côté atteste « rastẹtt, m. : eina amb tres pues de ferro i mànec de fusta, per arreplegar el fum [sic] ", et encore le diminutif " rastẹrẹ́t, m. : petita aixada per birbar el blat ". Néanmoins, n'oublions pas que le vocabulaire de Condò - né à Montcorbau - veut traduire, non pas la langue de son village natal, mais celle du terçon de Pujòlo, dans la partie la plus élévée du Val d'Aran : « els materials que l'integren reflecteixen principalment el parlar de Salardú » (Condò 1914 : 1). Cela jetterait

\footnotetext{
${ }^{2}$ Cette même application se retrouve en de nombreuses localités en Gascogne. Voir Schmitt (1934 : 90), qui cette fois-ci n'est pas mentionné par Coromines, ou encore l'ALG (III : 602; 'l'échine'), qui de son côté atteste la même formule à Saint-Gaudens (eth arrastèth dera (e)squia), à Saurat (le rastèl de l'[a]squena) ou à Auzat (le r[e]stèl de l'[a]squena). À ce sujet, voir encore Fossat $(1971: 229,345)$.

${ }^{3}$ Il précise : « A Puj i Ctro diferencien el rastèt del puat » ; " a Puj [...] rasteret no és més que un diminutiu de rastèt, un rascle petit ».

${ }^{4}$ Voir Carrera (2006 : 90). En fait, les définitions de Coromines (" eina [...] amb [dues o] tres pues de ferro", " herramienta [...] con tres púas de hierro ») sont très proches de celle de Condò (« eina amb tres pues de ferro $")$, pour ne pas dire identiques.
} 
le doute sur l'attribution de (ar)rastèth au centre du Val d'Aran si ce n'était le fait que I'ALC (I : 156; 'les arpies', à savoir 'les crocs à fumier') enregistre [er ras'tet] autant à Bossòst qu'à Vielha, où Griera a choisi Condò pour informateur.

D'après l'ALF (V : 1132), c'est bien arrastèth (ou, tout au plus, arr[e]stèth, ou encore un féminin arrastèra) le mot le plus répandu en Gascogne signifiant 'râteau'. ${ }^{5}$ En fait, il en va de même pour l'ensemble de la langue d'oc, car il y a des dérivés du latin RASTELLU (tels que rastèl, rastèu...) dans tous les départements situés en domaine occitan. ${ }^{6}$ La profusion de ce terme plaiderait à priori pour une présence aussi massive de arrastèth dans le Val d'Aran (en fait, les données de Coromines pourraient le laisser à penser), mais ce n'est pas le cas. D'après nos enquêtes, (ar)rastèth est le mot utilisé aussi bien à Canejan (sur ce point, nous sommes d'accord avec Coromines 1990 : 647) que dans tout le terçon des Quate Lòcs pour nommer un râteau quelconque, à savoir un instrument agricole dont la traverse munie de pointes est ajoutée à un long manche, quel que soit son nombre de dents. Cela veut dire que (ar)rastèth est également utilisé à Bossòst, mais aussi à Bausen et à Les, auxquels Coromines ne semble faire aucune référence (même si l'on sait qu'il a séjourné dans ce second village étant jeune). À Bausen et à Canejan, villages qui gardent la prothèse gasconne [a]- devant un R- (Carrera 2007 : 49 ; 2014 : 161-163; $2015: 80-81$ ) et qui connaissent le résultat -[t/f]<-LL' (Carrera $2008: 16-21)$, la prononciation normale de (ar)rastèth est [aras't $\mathrm{t} t$ /], alors qu'à Les et à Bossòst on entend [ras'tet] car, dans ce cas, la voyelle initiale s'est déjà effacée dans ces villages.

\footnotetext{
${ }^{5}$ Bon nombre de travaux corroborent ce fait. Entre autres : « arrastèt, arrestèt ; sm. - Râteau, créneau ; échine » (Palay, DBGM : 57) ; " ARRASTÈT, ARRESTÈT, râteau » (Lespy \& Raymond [1887] 1998: 42) ; " arrestèt, râteau ; fauchet " (Saint Gaudens ; Dupleich 1843 : 21) ; " ARRESTET, s. m., râteau " (Gers ; CénacMoncaut, [1863] 1993 : 12) ; "Arrestèth [arres'tet] m. râteau » (gascon maritime ; Moureau [1870] 1997 : 33) ; « râteau arrestet, restet » (gascon agenais ; Roques [1913] 1977 : 205) ; " râteau m. rastèth » (gascon toulousain ; Rei Bèthvéder 2004 : 239) ; " râteau m. (ar)rastèth » (Guilhemjoan 2003-2005, II : 306) ; " arrastẹc $\mathrm{m}$. 'râteau' < rastellu » (Lescun ; Bendel 1934 : 64) ; "Rastèt [sic], m. [...] Râteau " (Séronais ; Laurent 2002a : 152) ; " rrestęć [...] "râteau" < rastellu » (Bethmale ; Schönthaler $1937: 52$ ) ; Rastèth, m. [...] Râteau » (Aulus ; Laurent 2002b : 121). Voir encore Fahrholz (1931 : 83). C'est une forme bien attestée en gascon tout au long des siècles : voir, par exemple, Luchaire (1881: 191) ou Ginestet (2011 : 25). Pour I'application du terme (ar)rastèth au râtelier, voir Schmitt (1934:91) ou Cremona (1956:183). De manière inexplicable, le DECat (VII: 119) soutient que le " aranès arrastèt » signifierait 'râtelier', ce qui entre en contradiction avec ce qu'on peut lire chez Coromines (1990 : 285-286). Dans le Val d'Aran, dans ce cas, on dit (ar)rastilhèr.

${ }^{6}$ Outre le FEW (X: 94-98), voir les données rassemblées dans le THESOC (même s'il se sert d'une dénomination générale rastél [sic]), en provenance de plusieurs atlas linguistiques (s. v. râteau) : http://thesaurus.unice.fr/index.html.
} 
Dès Arres et Es Bòrdes, (ar)rastèth laisse sa place à pletera, que - comme annoncé nous allons aborder dans la section suivante ( $\S 3$ ). À notre connaissance (et contrairement à Coromines), hors des Quate Lòcs, (ar)rastèth ne refait surface que très sporadiquement. Là, sous forme de cas isolés, il se peut que ce mot désigne des outils munis de dents (apparemment en fer), mais non pas le râteau pourvu de nombreuses pointes. À Aubert (dans le terçon de Marcatosa, c'est-à-dire la zone qui a vu naître Jusèp Condò), notre informateur principal distinguait la pletera (le 'râteau', avec bon nombre de dents) du (ar)rastèth, qui à son tour n'aurait que quatre ou cinq pointes en fer et ressemblerait à 'ua horca virada' (ce qui nous fait penser aux définitions de Coromines et de Condò). ${ }^{7}$ Mais, bien que nos informateurs du centre et de la haute vallée aient été confrontés à des dessins de toute sorte d'outils agricoles à dents, la forme (ar)rastèth disparaît de (la plupart de) leurs réponses. Plusieurs d'entre eux vont même jusqu'à refuser ce mot, l'attribuant à d'autres villages aranais : à Es Bòrdes, on croit que (ar)rastèth est un terme de Bossòst (ce qui est juste) ; à Escunhau, ils assurent que ce mot est employé à Bagergue (qui apparaît comme le paradigme du parler aberrant) ; alors qu'à Bagergue on affirme ne l'avoir jamais entendu ; à Betren, presque la même histoire. On pourrait penser - en reprenant les mots de Séguy (1973 : 34) - que dans certains villages il n'y a pas de " disponibilité de premier rang " en ce qui concerne (ar)rastèth ; mais, en vérité, ce qui paraît le plus probable, c'est que ce mot n'est presque pas (ou presque plus ?) utilisé hors des Quate Lòcs. Si l'on fait abstraction des informations de Coromines et de Condò, il nous paraît assez significatif qu'une des toutes premières attestations de (ar)rastèth en aranais se trouve chez Barnils (1914 : 51), qui s'intéressait au parler de Canejan : « arastẹế RASTELLUM ». En fait, Coromines (1990 : 285) ne situe le

\footnotetext{
${ }^{7}$ Cette utilisation spéciale - ou plutôt spécialisée - de (ar)rastèth rappelle celle de (ar)rascleta ou - peutêtre - (ar)rasclet hors de l'aire de (ar)rascle, 'râteau'. Voir ci-dessous (§ 4, note 17). En fait, le (ar)rastèth $d^{\prime}$ 'Aubèrt semble être le même instrument que le arrastèth deth hiem(s) de Tramezaygues ou de SaintPlancard qui se trouvent dans I'ALG (II : 310). C'est un outil à manche oblique (ou plutôt doté d'un manche à angle droit) avec quatre dents en fer, parfois appelé rastrilha ailleurs en Val d'Aran. Par contre, le (ar)rastèth de Quate Lòcs pourrait être relié à la situation la plus fréquente en gascon. Quoique la plupart des dictionnaires se contentent de définir (ar)rastèth comme 'râteau', si l'on jette un coup d'œil - par exemple - aux photographies d'Arnaudin, on s'aperçoit qu'autant le « râteau " que le " [c]roc à fumier à quatre dents en fer » sont nommés arrestèth en landais (Boisgontier 2001 : 61-62).
} 
dérivé "Arrasterà [sic] 'rampinar' " qu'à Sant Joan de Toran. ${ }^{8}$ Lui-même ajoute une observation très intéressante sur ce verbe, tirée des documents inédits de Condò : " Condò l'equipara a rasclà [sic] com a propi de $Q L$ ». Ces allusions au plus nord-occidental des terçons aranais - qui, à première vue, sembleraient sans importance - ne sont probablement pas dues au hasard, car elles s'accordent avec ce que nous avons pu constater sur le terrain : le terçon des Quate Lòcs - tout comme le Bavarthès ${ }^{9}$ - se sert du terme le plus répandu en gascon pour désigner le râteau ((ar)rastèth), alors que le reste du Val d'Aran, en règle générale, utilise d'autres dénominations.

\section{Pletera, 'râteau'}

Coromines (1990 : 647) a prêté beaucoup d'attention à ce mot, au point de lui conscarer une entrée longue de vingt-huit lignes. D’abord, ce linguiste rapporte les définitions des documents inédits de Condò et restreint l'étendue de pletera au centre de la vallée :

PLETÉRA [sic] f. « rascle amb pues de fusta o ferro, per arreplegar l'herba o esbarriar la terra » Condò (com a particular del CV) [...] " rampill o rascle id. id. ", amb un c[r]oquis dibuixant una eina amb mànec més llarg que el travesser, implantat al cap, i en aquest clavades deu pues. Amb pron. plẹtẹ́ra unànime i com a nom del rascle per a herba, el compr. a 18 p., tots dels tres terçons centrals, des d'Aes, Bo i Vm fins a Esc i Clh.

Puis, Coromines se penche sur son étymologie et, en même temps, se plaint du fait que les romanistes sont passés à côté de cette dénomination aranaise :

\footnotetext{
${ }^{8}$ En réalité, il est utilisé ailleurs dans les Quate Lòcs. Nous l'avons entendu à Les, par exemple.

${ }^{9}$ D'après Heyns (1938: 75), 'Rechen' (c'est-à-dire 'râteau') est « arastęč » à Fos, mais « āráskle » à Cierp. Néanmoins, cette dernière attribution est douteuse du moment que, deux pages plus loin, ce dernier mot étonnamment, sans la prothèse - devient 'Egge' à Fos (Heyns 1938 : 77 ; cfr. ci-dessous, § 4).
} 
Del II. vg. *APPLǏC(Ĭ)TŌRIA, deriv. de APPLICARE en el sentit de 'aplegar, arreplegar, recollir', forma del tema de supí-participi, que és d'esperar, atesa la forma PLǏCITUS que ja és clàssica en aquest tema de PLICARE. [...] Encara que ja ho vaig establir en la meva tesi doctoral de $1928-31,{ }^{10}$ tots els romanistes (fins els estudiosos del gascó) han seguit fent orelles de mercader a l'antigor i l'existència d'aquest mot, que té la " desgràcia » de conservar-se només en l'Aran : els sancta sanctorum de la llengua d'oc.

Coromines (ibidem) renvoie le lecteur à son dictionnaire étymologique catalan (" Detalls en PLEGAR, DECat "), où nous n'avons pu déceler aucune référence à la forme aranaise. Quoi qu'il en soit, l'étymologie proposée est satisfaisante sur le plan phonétique, car l'aphérèse est bien possible et que la réduction de la diphtongue [ej] dans ce contexte-là n'a rien d'extraordinaire en aranais (cfr. Bec 1968 : 105-113) : era *(a)ple(i)tera > era pletera. La forme pletera se trouve déjà chez Soler i Santaló ([1906] 1998 : 356) : "pletera, rascle ». Mais ce mot figure aussi dans le répertoire de Condò (1914 : 23 ; " plẹtẹra, f. : rascle, eina amb pues de fusta per arreplegar l'herba i la palla »), ce qui prouve - à nouveau - que le poète de Montcorbau a recueilli dans son vocabulaire des mots n'appartenant pas à Salardú. ${ }^{11}$ Ce qui est assez symptomatique (et s'accorde avec les données de Coromines), c'est que le peu d'occurrences de pletera fournies par la bibliographie provient notamment d'auteurs du centre du Val d'Aran (exception faite du catalan Soler i Santaló). D’une part, l'avocat de Vielha Casimiro Ademá (1966 : 57), qui recueille "Pletera = rastrillo de madera». ${ }^{12}$ D'autre part, Vergés $(2009: 185,371)$, pour

\footnotetext{
${ }^{10}$ Voir Coromines (1931: 97). Là, le mot pletera, 'rastro para recoger hierba o paja' est associé a cinq villages aranais : Vilamòs, Montcorbau (Condò ?), Vilac, Gausac et Escunhau. Par contre, " en Pujolo y Quate Locs, aráskle o arastęć ». Comme signalé plus haut (§ 2), l'attribution aux Quate Lòcs de cette acception se base uniquement sur Sant Joan de Toran (voir Coromines $1931: 11$ ).

${ }^{11}$ Condò (1914 : 23) atteste encore le verbe "rẹplẹterá, v. arreplegar l'herba amb el rascle de fusta ", de même qu'un substantif « rẹplẹlẹra [sic], f.: rascle de fusta ». Coromines (ibidem) reprend ce dernier mot et le transforme en " repletẹ́ra », mais il n'a pas pu en vérifier l'existence : " un substantiu [...] que no tinc compr. ". Au cours de vingt ans d'enquêtes, nous ne l'avons pas non plus entendu. Bien probablement, il s'agit d'une erreur. Le verbe (ar)repleterar se trouve à Vielha selon I'ALC (VII : 1129 ; 'rasclar'), alors que ersar (sur lequel nous reviendrons plus loin, § 6) est repéré à Bausen, Vilac et Es Bòrdes, (ar)rasclar à Gessa, à Arties, à Aubèrt, à Bossòst et à Les (cfr. §4), sauclar à Aubert, et arrest[a]rar à Canejan et à Les (probablement pour arresterar).

${ }^{12}$ Et encore le dérivé « Repleterà = rastrillar » (Ademá $\left.1966: 58\right)$.
} 
qui pletera est un synonyme de arrastèth et signifie 'rastrillo', 'rascle' et 'râteau'. Ce n'est pas par hasard que Campá (1983 : 37) se trompe sur la transcription phonétique de pletera (sa voyelle tonique n'est jamais mi-ouverte), car a Bossòst - son village natal - ce mot n'est pas du tout utilisé. De son côté, Krüger ([1939] 1996 : 348) fait mouche en situant pletera à Es Bòrdes.

En effet, en ce qui concerne l'étendue de pletera, on doit valider les informations de Coromines, car nous l'avons relevé à Arres, à Vilamòs, à Es Bòrdes (" eth rastèth ei era pletera, lo que passe qu'en Bossòst en diden rastèth »), à Aubèrt (" entà arrepleterar era èrba "), à Vielha, à Betren et à Escunhau. II s'agit partout d'un râteau muni d'un certain nombre de dents. À Arties, où l'on utilise déjà (ar)rascle (§ 4), l'un de nos informateurs précisait que cet instrument s'appelle pletera à Escunhau. Dans le terçon des Quate Lòcs, on sait bien que pletera est employé " plus haut ", même si l'on a souvent du mal à en établir l'étendue : " ac diden per aquiu ensús » ('là-haut' ; Bausen) ; « en Pujòlo, de Vielha tà naut » ('à Pujòlo, en amont de Vielha'; Bossòst). Rien de nouveau sous le ciel : au Baish Aran, les mots considérés comme anormaux sont souvent attribués au Naut Aran. Et inversement.

\section{4. (Ar)rascle : 'râteau' ou 'herse' ?}

Apparemment, sous l'entrée pletẹra de sa thèse de doctorat, Coromines (1931: 97) attribue ắáskle à Pujòlo, " rastro para recoger hierba o paja ". Ce mot serait un synonyme de arrastèth et de pletera. ${ }^{13}$ Mais si l'on regarde l'entrée consacrée à arastęć, les différences sont sensibles. Là, ăắskle est toujours présenté comme un synonyme de arrastèth (plus spécifiquement, du arrastèth relevé à Sant Joan de Toran ; " rastro para recoger la hierba y otros usos ») et de pletera (terme propre à " Castiero, Marcatosa y Lairissa »). Mais, cette fois-ci, arrascle n'appartient plus à Pujòlo, mais à Bausen : « aráskle [Ba] (como en cat.) " (Coromines 1931 : 11). Ce fait (il s'agit d'une antilogie pas du tout isolée chez lui ; cfr. Carrera 2006 : 97-98) est d'autant plus étonnant que ces deux entrées

\footnotetext{
${ }^{13}$ Voir note 10.
} 
ont fusionné dans la monographie de 1990, sous le lemme rascle. Là, ce mot - qui a perdu sa prothèse -, toujours synonyme de (ar)rastèth et de pletera, est étiqueté comme un terme propre à Pujòlo et, en même temps, à Bausen :

RASCLE m. = cat. RASCLE, terme particular de Puj (pletera RV, i cf. rastèt) : és tot de fusta si serveix per a la palla i l'herba, i de ferro si és per a les pedres i terra [...] ; Mg, Bag, Sal, Tr, Uny, Art, i de nou Ba.

Remarquons que cette nouvelle formulation pose de nouveaux problèmes, car pletera est désormais un mot du $R V$ - le reste du Val d'Aran -, ce qui n'est pas le cas et qui se heurte à ce qu'on trouve sous l'entrée consacrée à ce mot dans le dictionnaire aranais de Coromines (1990: 697 ; cfr. § 3) qui, par ailleurs, croit que le verbe primitif (ar)rasclar pourrait être justifié ainsi : «El més simple seria admetre rascar > rasclar amb repercussió de líquida, forma consolidada per la influència d'asclar ${ }^{14}$ (DECat VII : 132 ; s. v. raure). Mais revenons-en à l'étendue et au signifié de (ar)rascle. D’après nos données, (ar)rascle, prononcé ['raskle], désigne un râteau quelconque autant dans le terçon d'Arties e Garòs que dans celui de Pujòlo, c'est-à-dire dans tout le Naut Aran. Selon nos informateurs, c'est le même outil qui est appelé ailleurs pletera. ${ }^{15}$ En conséquence, l'isoglosse qui sépare pletera de (ar)rascle est à situer à la limite des communes de VielhaMijaran et de Naut Aran. Cela s'accorde avec les données de Fritz Krüger ([1939] 1996 : 347-348), pour qui « ráskle » est un « rastrillo con numerosas púas de madera » dans des villages tels que Montgarri et Salardú, et un « rastrillo [...] provisto con dientes fuertes y

\footnotetext{
${ }^{14}$ À propos de la question étymologique, voir entre autres Bloch \& Wartburg (1975: 529$)$, le FEW (X : 79, 84 ; *RASCLARE aussi) ou le DCVB (IX : 152; *RASTULARE). Coromines (DECat VII : 132) conteste *RASTRULARE « car no sols fóra de mal justificar morfològicament una base verbal així, partint només d'un substantiu llatí, sinó que és absolutament rebutjable des del punt de vista fonètic: admesa i tot la possibilitat de la dissimilació eliminatòria de la segona -R-, és evident que, en condicions tan secundàries $\mathrm{i}$ tardanes, de cap manera no s'hauria pogut produir el canvi de ST'L en -SCL-, que és solament de data arcaica en II. vg. » (DECat : ibidem).

${ }^{15}$ À Bagergue on attribuait pletera à Les. D'après I'un de nos informateurs d'Arties, en revanche - nous y avons déjà fait allusion : $\S 3$-, ce mot serait déjà en usage à Escunhau (c'est bien le cas). À Garòs, on distinguait le (ar)rascle dera èrba et le (ar)rascle dera palha.
} 
bastante largos » à Gessa (Krüger [1939] 1996 : 233). ${ }^{16}$ Montgarri - aujourd'hui inhabité -, Salardú et Gessa se trouvent tous en Naut Aran. ${ }^{17}$

À quoi tient l'attribution à Bausen de (ar)rascle 'râteau' qu'on peut lire chez Coromines ? Bien probablement à une confusion entre les désignations du râteau et de la herse, une sorte de grille à grosses pointes qui était traînée sur le sol par un attelage. Non seulement cette confusion pourrait être alimentée par le signifié du terme (ar)rascle en haut-aranais, mais aussi par le fait que rascle désigne également le râteau dans une partie importante du domaine linguistique catalan, à commencer par des villages proches du Val d'Aran appartenant au Pallars ou à la Ribagorce. ${ }^{18}$ De ce fait, ayant entendu arrascle à Bausen (où en vérité ce mot sert à nommer la herse), Coromines lui aurait attribué tout naturellement la valeur de 'râteau'. Toujours à ce sujet, signalons quand même que rascle peut nommer aussi la herse en catalan et que cet emploi est plus répandu que le

${ }^{16}$ Dans ce dernier cas, Krüger renvoie à une photo où l'on peut voir un râteau à six dents (photo 33). En vérité, sur le plan morphologique, il s'agit d'un râteau du même genre que le précédent. Seule différence : ce dernier possède jusqu'à treize pointes (photo 23).

${ }^{17}$ Ajoutons qu'il existe un dérivé (ar)rasclet qui semble plus courant en Naut Aran qu'ailleurs. D'après Coromines, " [e]l rasclet es diferencia del rascle perquè és el que s'usa per netejar la terra Gar, mentre que el rascle és per a la herba a Uny, i a Aub es torna el nom del xadet » (Coromines 1990 : 285), c'est-à-dire du (ai)shadet, une sorte de houe. À notre connaissance, alors que le (ar)rascle désigne génériquement le râteau, (ar)rasclet peut se rapporter plutôt à un râteau en fer (par exemple, à Arties), utilisé pour « es graves o es pèires " ('le gravier et les cailloux' ; Salardú), avec un nombre réduit de dents. Au vu de la réponse d'Aubèrt obtenue par Coromines et du fait que Casimiro Ademá (1966: 58) atteste rasclet 'rastrillo de hierro' (ou même après avoir constaté que (ar)rastèth pourrait faire son apparition en dehors de la zone où il désigne le râteau grâce à des applications spécifiques; cfr. ci-dessus, $\S 2$ ), il ne faut pas exclure que (ar)rasclet dépasse - ou ait dépassé - l'aire de (ar)rascle pour désigner des outils en fer proches du râteau ou même dépourvus de dents. Ce qui nous paraît évident, en tout cas, c'est que (ar)rascle, hors du Naut Aran, ne peut jamais désigner génériquement le râteau. Quoi qu'il en soit, le dérivé du verbe (ar)rasclar le plus répandu en aranais est sans doute le féminin (ar)rascleta, une sorte de raclette à pétrin. Nous avons relevé ce mot dans plusieurs villages aranais (Bausen, Canejan, Les, Arties). Il s'agit de l'instrument aussi appelé (ar)rasqueta (<(ar)rascar ; Escunhau, Arties, Gessa, Bagergue), (ar)raspeta (< (ar)raspar ; Garòs) ou encore (ar)rasera (< (ar)rasar + -ORIA ; Bossòst, mais dans ce cas ce grattoir est surtout utilisé pour nettoyer la cheminée; à Es Bòrdes la voyelle tonique est $[\varepsilon]$ en raison d'une analogie avec les nombreux mots contenant -ARIA ; sur ce terme, cfr. Coromines [1990: 285], même si ce linguiste soutient qu'il est « pertot pronunciat amb é tancada » en Val d'Aran).

${ }^{18}$ Voir l'ALDC (IV : 842 ; 'El rampí) : rascle figure dans des localités telles que Alins, Senet (seconde réponse) ou Les Paüls (ici, c'est rasclle, avec la palatalisation de la latérale propre au ribagorçan). Cette désignation se poursuit vers le sud (rascle est même utilisé à Valence et à Alfafar), vers l'est (rascle en tant que deuxième réponse à Corneilla-de-Conflent et à Montauriol, dans les Pyrénées Orientales, et rascle d'entaular à Perpignan) et vers l'ouest (benasquais rascllo ; cfr. Ferraz 1934 : 92 ; Ballarín 1978: 412 ; et, encore une fois, Krüger, [1939] 1996: 348). D’après le DCVB (IX : 152), rascle, dans sa deuxième acception, désigne un « [i]nstrument compost d'un mànec molt llarg que a un extrem porta entravessada una post guarnida de pues de fusta o de ferro, que serveix per a arreplegar herba o palla (Pallars, Conca de Tremp, Pla d'Urgell, Camp de Tarr., Gandesa, Tortosa, País Valencià) ». 
précédent. Il est également connu des modalités voisines du gascon (mais ignoré du hautaranais, comme nous aurons l'occasion de le voir). ${ }^{19}$

Rien de surprenant non plus à ce que (ar)rascle désigne la herse en gascon. D'après I'ALG (II : 272), (ar)rascle sert à nommer cet instrument un peu partout dans les Pyrénées : il est utilisé en Béarn, à Riscle (Gers), dans presque tout le département des HautesPyrénées, ${ }^{20}$ dans la plupart du Comminges, dans la partie la plus occidentale du Couserans (Castillon et Bethmale), ainsi qu'à Saverdun (Ariège). Selon I'ALF (III : 689), rascle serait encore en usage à Fanjeaux (Aude) et un terme errascle se trouverait même à Montastruc-la-Conseillère (Haute-Garonne, au nord de Toulouse). ${ }^{21}$

C'est cette même dénomination, (ar)rascle, qui évoque la herse à Bausen, mais aussi dans une partie non dédaignable de la vallée, même si Coromines n'en était pas au courant. Heyns (1938 : 75) avait déjà recueilli « Egge ráskle Ls Bo Fo », c'est-à-dire à Les, à Bossòst et à Fos. Mais l'aire de ce mot désignant la herse est beaucoup plus importante en Val d’Aran. En fait, elle couvre en gros la moitié de la vallée et s'arrête pas loin du point où (ar)rascle passe à désigner le râteau. À Canejan et à Bausen, on garde la prothèse

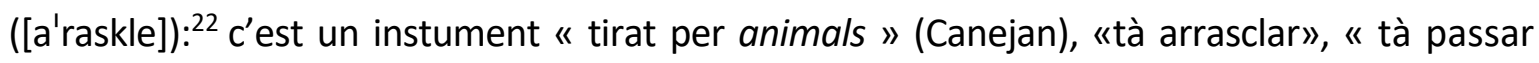
per un camp "(Bausen). Dès Les, la prothèse disparaît (['raskle]). Nul doute que les définitions font toujours référence à la herse : " tà remòir era tèrra " (Bossòst) ; " ua causa grana que tire ua bèstia " (Es Bòrdes). Ce mot est toujours utilisé à Vielha, mais il paraît s'estomper aussitôt, car à Betren nous ne l'avons plus entendu. C'est le seul endroit aranais où l'on n'ait pas pu obtenir de dénomination pour la herse.

\footnotetext{
${ }^{19}$ Voir l'ALDC (IV : 760), carte 'El rascle'. Le titre de la carte est déjà très révélateur. Remarquons que rascle 'herse' est la première réponse à Esterri d'Àneu, à Alins ou à Taüll, à proximité du Val d'Aran.

${ }^{20}$ Apparemment, la moitié septentrionale de ce département est plutôt polymorphique : (ar)rascle et èrsa.

${ }^{21}$ Rascle figure chez Alibert (1997 : 580) en tant que - entre autres - 'herse de labour'. Ce mot et cette acception ont déjà été recueillis par une de ses sources les plus importantes, le dictionnaire de Mistral (TDF, II : 703) : « RASCLE, ARRASCLE (g. [gascon]) [...] s. m. Herse de labour [...] ; coupe-pâte [...] ; ondée de pluie violente et courte ". Pour d'autres formations et applications parallèles à (ar)rascle en galloroman continental, toujours en relation plus ou moins étroite avec racler/rasclar, voir le FEW (X: 80).

${ }^{22}$ Même si Heyns 1938: 75) atteste [r]- à Fos et que Krüger ([1939] 1996 : 107) écrit "St. Béat ráskle ", la réalisation [a'raskle] est générale en Bavarthès d'après nos enquêtes (même à Boutx, la localité la plus éloignée de la Garonne). Cela s'assortit avec le arrascle d'Arguenos de l'ALG (II : 272) et avec la forme de la Barousse : " arráscle, [arrascle] s. m. Herse ", et encore " arasclá, [arrasclar] v. Herser » (Soulé-Venture ; Le Nail \& Eygun 2015 : 30). À Castillon et à Bethmale, en Couserans, le manque de la prothèse est la solution normale, contrairement à ce qui arrive en commingeois (voir Bec 1968 : 180-181).
} 
En définitive : le mot (ar)rascle peut désigner deux instruments différents en aranais. D'un côté, c'est le râteau dans la haute partie de la vallée, ce qui est à mettre en relation avec les localités catalanes qui se servent de ce terme pour nommer cet outil-là (dont certains avoisinent le Val d'Aran). D'un autre côté, (ar)rascle désigne la herse sur un espace qui va des Quate Lòcs jusqu'au centre de la vallée. Cet emploi, également connu en catalan, ne peut pas être détaché de l'utilisation de cette même forme dans la plupart du gascon pyrénéen. En Val d'Aran, cette dernière aire ne se superpose pas à la précédente.

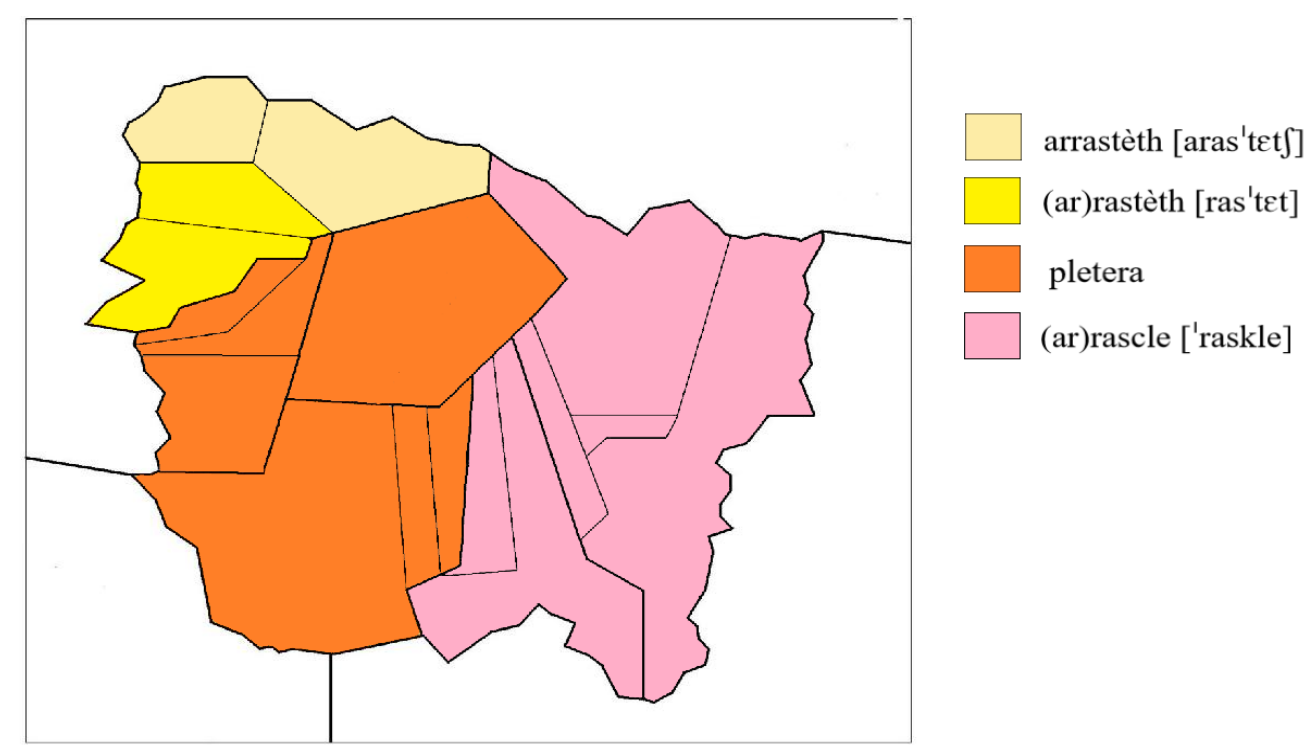

Carte 2. Râteau

\section{Carràs, 'herse'}

Sur le territoire qui s'étend de la frontière de Pònt d'Arrei jusqu'à la limite de la commune du Naut Aran, nous n'avons pu déceler - outre Betren - qu'une localité échappant à la règle de l'omniprésence de (ar)rascle 'herse' : Escunhau, tout près de la frontière haut-aranaise. Là, nos informateurs nous ont fourni la forme carràs. Ce terme, 
dont l'étymologie a été étudiée par Coromines (DECat, II : 589-590), ${ }^{23}$ est bien connu en occitan, où il peut désigner un 'faisceau de bois ou de branchages' ou une 'grappe de raisin' mais, en même temps, des radeaux ou des assemblages de branches ou de pièces de bois, éventuellement roulés sur le sol (FEW, Ila : 428, s. v. carrus ; TDF, I : 477). De ce fait, on comprend bien qu'il puisse aussi désigner des traîneaux (tel est le cas de certaines localités de l'Ariège comme Auzat, Mérens-lès-Vals ou Saurat ; ALF, VI : 1322; ALG, II : $350),{ }^{24}$ et que ce glissement sémantique l'ait amené jusqu'à signifier la herse dans certaines zones du domaine occitan. Mistral (ibidem) signale déjà que carras, caras peut avoir le sens de " traîneau, herse ". Ce deuxième signifié paraît bien enraciné - par exemple - en Rouergue, car là corrás ou escorrás [carràs, escarràs] peuvent aussi servir à désigner la herse (Vayssier 1879 : 111, 224, 570). L'ALF (III : 689) atteste carràs à Rieupeyroux, à Calmont (Aveyron) et encore à Valderiès (Tarn), et encore escarràs à Laguépie (Tarn-etGaronne) et à Gaillac (Tarn).

Justement, en aranais, la première définition de carràs apportée par Coromines (1990 : 390) est " munt de branques espinoses ». Mais, parmi ses applications, remarquons celle de "'lligall d'espinalls amb pedres a damunt, que s'arrossega pel prat, per escampar-hi un pilot de fems' [...] ; arrossegat per muls ", à propos de laquelle le linguiste catalan renvoie à Krüger qui, de son côté, atteste « V. de Arán sept. karrás "haz de troncos delgados, que los mulos arrastran por el suelo" » (Krüger [1945] 1997 : 200). Cette information est attribuée à Heyns, qui recueille le dérivé «kā̄asés» (Heyns 1938 : 152), dont Coromines s'occupe aussi ("Carrassè [sic] m. 'raier' "; ibidem) et sur lequel nous avons eu l'occasion de nous pencher en détail tout récemment (Carrera sous presse). Apparemment, en tant que 'herse', le mot carràs n'avait pas été noté jusqu'ici en Val d'Aran. À Melles, en Comminges, une sorte de petite herse est appelée carrasson.

\footnotetext{
${ }^{23}$ Voir également le DECH (1 : 893-894).

${ }^{24}$ Voir encore Fahrholz (1931: 141) ou même Palay (DBGM : 206), qui donne "[r]éunion de choses attachées ou enfilées à la façon d'un chapelet " (c'est la même définition apportée par Rohlfs [1977 : 64] pour le pays de Barèges), mais aussi « [e]n Ari. [Ariège] traîneau ».
} 


\section{Les désignations haut-aranaises de la herse : èrsa et arpèga}

La plupart des villages haut-aranais désignent la herse par le moyen d'un mot inconnu des autres localités de la vallée. Il s'agit de arpèga, que Coromines ne semble pas avoir entendu au cours de ses enquêtes en Val d'Aran. Ainsi, arpèga est un instrument qui sert " tà estarrossar » ['briser les mottes'] (Tredòs), c'est le même outil qui vers le centre de la vallée est appelé (ar)rascle (Bagergue). Également connu à Salardú et à Gessa, son aire se termine plus à l'est. À Garòs le nom est connu, mais on paraît lui préférer èrsa, le seul terme admis à Arties (où, d'un autre côté, un de nos informateurs précisait que arpèga appartenait probablement au terçon de Pujòlo, ce qui est juste). Quoi qu'il en soit, tout porte à croire que èrsa est un terme plus implanté à Arties qu'à Garòs, même si cela peut paraître, à première vue, un peu choquant. Arties pourrait être la localité à partir de laquelle èrsa rayonnerait. Mais tout cela n'est qu'une hypothèse à valider. Étonnamment, le lexique de Besson \& Besson (2005: 81), originaires d'Arties, recueille "rasclé, m. : grand râteau des prés ", et non pas les termes èrsa et arpèga. Mais n'oublions pas que ces auteurs mélangent des formes en provenance d'Arties et d'autres qui ont été puisés notamment - chez Coromines. ${ }^{25}$

La vérité est que, à notre connaissance, il ne paraît y avoir qu'une seule recherche qui atteste le mot arpèga. Dans un travail s'intéressant au parler de Bagergue, dont l'auteur était originaire de Garòs, on peut lire ce qui suit : "Arpèga (hèrsa) : appareil pour émotter les petits blocs de terre dans les champs labourés » (Barès 2001 : 91). L'auteur se contente de signaler que ce mot ne se trouve pas chez Coromines (1990) et, dans la mesure où il apporte des transcriptions des enquêtes, nous fournit quelques explications supplémentaires sur ce terme, qui désigne incontestablement la herse. ${ }^{26}$

Bien que confiné à la partie la plus élevée en Val d'Aran, arpèga n'est pas un mot isolé en gascon. D'après l'ALG (II : 272), il est utilisé dans presque tout le département des Landes et dans plusieurs localités orientales de la Gironde, mais aussi à Biarritz. Selon

\footnotetext{
${ }^{25}$ Parfois, ce fait devient très évident, car ce vocabulaire répertorie des formes très locales n'appartenant pas à Arties, dont l'origine est incontestablement Coromines (cfr., par exemple, "aranyo » [aranhon, forme propre au canejanais] à côté de « granyon » [granhon] ; Besson \& Besson 2005 : 102).

${ }^{26}$ « Era arpèga, jo n'è dues, ena tèrra. Tà passà-la [sic] quan avies semiat es truhes, ath cap de quinze dies sortive un saucle petit, praua [sic] de tu, non te calie sauclar ! I passaves aquerò, aquerò te quedave límpio [sic], un dia de calor aucive eth saucle. Vosati didetz era hèrsa, nosati era arpèga » (Barès 2001 : 91).
} 
I'ALF (III : 689), il y aurait encore des masculins comme arpèc à des endroits tels que Targon et Saint-Côme (Gironde). Le mot relevé en aranais figure déjà chez Moureau ([1870] $1997:$ : 32) : « Arpèga [ar'peyə] $f$. herse » (juste avant le verbe arpegar, 'herser'). Mais aussi chez Arnaudin (Boisgontier 2001 : 47) : « arpégue <arpèga> s.f. Herse » (cette fois-ci, juste après « arpega <arpegar> [è] v. tr. Herser »). Palay (DBGM : 59), qui à son tour atteste aussi le verbe arpegar (" arpegà; v. - Herser ; par anal. griffer »), le considère également comme un mot landais : "arpègue ; arplégue (L.) ; sf. -Herse, griffe aratoire ». En fait, ce terme - et encore le verbe « ARPEGA » - se trouve même chez Lespy et Raymond ([1887] $1998:$ 40), mais sans aucune marque géographique : « ARPÈGUE, herse ».

Nul doute que arpèga est un déverbal du verbe *HIRPICARE plutôt qu'un résultat de HIRPEX, -ICIS (qui donne le français herse et l'italien erpice). Telle est, à ce qu'il paraît, la position de Wartburg (qui place les formes précédentes [" bearn. land. arpègue Teste arpégue »] sous l'entrée *HĬRPǏCARE ; FEW, IV : 433) et de Ronjat (GIPP, II : 251), qui signale : " les tipes [sic] suiv. sont refaits sur le verbe "erser" qui, suivant les traitements dialectaux de lat. -ca- après voy. et de rom. $e+r+$ consonne [...] est er- $\sim$ arpega $\sim$-peia $\sim$-pia(r) : masc. arpegue $\sim$-pègue $\sim$ pèc (-è- sans doute par alt. vocalique analogique) très répandu dans les dép. Gironde et Landes ».

Si l'on se concentre sur les faits gascons (et que l'on laisse de côté d'autres déverbaux parallèles qui se trouvent en domaine occitan tels que àrpio, èrpio, erpeia, erpia...), ${ }^{27}$ un fait important est à remarquer : le terme haut-aranais arpèga peut être relié à son homologue landais mais, tout d'abord, il ne peut pas être détaché de ce qu'on peut observer à plusieurs endroits du département voisin de l'Ariège. Bon nombre des réponses ariégeoises de I'ALG (II : 272) attestent des désignations de la herse liées soit à HIRPEX, soit - plutôt - à HIRPICARE : èrp (Saurat), èrpe (Auzat, Le Mas-d'Azil), èrpet (La Bastide-de-Sérou), erpec (Lescure). Ajoutons que l'ALG atteste encore arpegar à Castillonde-Couserans ('gratter' ; ALG, II : 451, carte 's'épouiller') et à La Bastide-de-Sérou ('herser' ;

\footnotetext{
${ }^{27}$ Pour lesquels nous renvoyons surtout aux données répertoriées par Wartburg (FEW IV : 433), Mistral (TDF I : 967) et Ronjat (GIPP II : 251). N'oublions pas, à ce propos, que Coromines (DECat I : 402) suggère qu'il pourrait y avoir eu un croisement de certains dérivés du francique HARPA et du latin HIRPEX qui aurait touché des formes telles que le catalan àrpies, arpiots et arpelles, mots désignant des instruments agricoles à pointes. N'oublions pas non plus que, parmi les acceptions de arpa en gascon, il existe celle de 'herse' (voir, entre autres, Palay DBGM : 54).
} 
ALG, II : 253, carte 'briser les mottes') ${ }^{28}$. Fahrholz (1931: 80), de son côté, rassemble jusqu'à neuf exemples qui, dans plusieurs cas, viennent élargir ce répertoire, même un substantif dérivé de HIRPICARE par le moyen d'un suffixe :

[ęrp] Verdun, Saurat-Tal, [ęrpẹ] Axiat, Bouan, Le Pech ; [arpét] Appy ; vgl. erpi, erpe, arpe (langued.), 'herse' [...] 'Egge' [...] - [erpegadú] Senconac, Unac; zum Verbum [arpẹgá] 'eggen'

Ce genre de formes continuent vers l'est. L'ALF (III : 689) atteste èrpet non seulement au Mas-d'Azil, mais aussi à Crampagna (Ariège) et à Rivel (Aude). ${ }^{29}$ Cela peut être mis en relation avec les formes du type erpo et èrpol (< HIRPICE) qui se trouvent en Catalogne Nord (DECat III : 423 ; ALDC IV : 760 ; DCVB V : 142). En conséquence, tout semble indiquer que, dans un vaste espace pyrénéen allant du Naut Aran à la Catalogne Nord (en passant par l'Ariège et par certains endroits du département de l'Aude), HIRPEX et HIRPICARE auraient mieux résisté qu'ailleurs. On est même tenter d'imaginer que certains parlers couserannais, qui feraient le lien entre le Naut Aran et des endroits ariégeois situés plus à l'est, auraient connu ce genre de formes à un moment donné, avant de céder à la pression du mot (ar)rascle, prépondérant en gascon pyrénéen. Mais ce ne sont que des suppositions.

Et que dire de èrsa ? En fait, il s'agit du mot le plus répandu en occitan. En Gascogne, d'après l'ALG (ibidem), son aire couvre la plupart du département du Gers, de nombreuses localités en bord de Garonne en aval de Martres-Tolosanne et une bonne partie de la Gironde. En situation de polymorphisme, ce terme fait concurrence à d'autres formes (notamment (ar)rascle) dans la moitié septentrionale des Hautes-Pyrénées, à des endroits tels que Arreau ou Tramezaigues et même en Haut Comminges (Saint-Gaudens, Bourg d'Oueil). En règle générale, ce sont les zones les plus proches de la Garonne et de Toulouse celles qui se servent de ce mot, ce qui nous paraît assez symptomatique. Même

\footnotetext{
${ }^{28}$ À Castillon comme à Bethmale le mot désignant la herse est (ar)rascle, le terme le plus répandu en gascon pyrénéen, d'après I'ALG (II : 272). Ce sont les localités les plus orientales - exception faite de Saverdun - qui se servent de cette dénomination dans l'atlas gascon. Cfr. ce que nous avons exposé cidessus (§ 4).

29 II semblerait que ce mot soit absent du répertoire de Lagarde (1991).
} 
si èrsa < HIRPICE peut se défendre sur le plan évolutif, ${ }^{30}$ la disposition géographique de ce mot plaide évidemment pour une origine française de cette forme. C'est la position de Ronjat (GIPP, II : 251), qui signale « la capricieuse répartition géographique [sic] du tipe ers- " - ajoutons : hors de Gascogne - ainsi que " son absence en vpr. " comme des éléments qui « portent à i [sic] voir un emprunt au fr. ». Jean Séguy (1956: 54), de son côté, s'est servi du syntagme « mot français » en faisant allusion à ce terme. La réalisation ['her]- attestée à de nombreux endroits de Gascogne ne paraît nullement s'opposer à ce que nous venons de formuler, car une immixtion de hèr 'fer' nous paraît plus que vraisemblable.

Le fait que èrsa, dans le Val d'Aran, se trouve loin de la frontière politique pourrait être vu comme un obstacle à cette origine française. Jusqu'ici - à l'exception du travail mentionné plus haut - la seule attestation aranaise liée à ce mot, c'est le verbe ersar, relevé par l'ALVA (1111; 'rasclar') à Bausen, à Es Bòrdes et même à Vilac. Mais ces données de Griera - comme tant d'autres - ne nous paraissent pas crédibles (Carrera 2004 : 6-14). En outre, èrsa peut faire son apparition à des endroits très méridionaux et plus ou moins isolés, détachés de la vaste aire garonnaise-toulousaine où ce terme est prépondérant. C'est le cas, par exemple, de Couflens (ALG : ibidem). En fait, alors que le luchonnais se sert de (ar)rascle, ersa resurgit dans des localités telles que Cerler, Eressué ou Les Paüls, au sud des Pyrénées (ALDC : ibidem). Peut-être qu'il y a des faits presque anecdotiques (ou des faits matériels; en tout cas, des faits locaux) qui nous échappent, tels que la diffusion des herses métalliques, qui aurait contribué à la diffusion du francisme d'après Séguy (ibidem). ${ }^{31}$ On ne saurait exclure que la présence préalable d'un dérivé autochtone de HIRPEX, proche du francisme herse sur le plan formel, ait favorisé l'enracinement de cette forme, dans la mesure que èrsa, en aranais, se trouve juste à côté des dérivés de HIRPICARE. Quoi qu'il en soit, le plus probable est qu'un francisme est venu s'installer à côté de arpèga. Tout d'abord, il aurait réussi à s'établir à Arties. De

\footnotetext{
${ }^{30}$ Dans ce cas, il faudrait écrire èrça. En ce qui concerne le masculin èrce relevé autant en Gironde que dans les Pyrénées, cette hypothèse paraît vraisemblable, plutôt plausible.

${ }^{31}$ Séguy remarque que " le mot français s'applique de préférence aux types métalliques récents en usage dans la zone de grande culture ».
} 
là, il pourrait avoir atteint Garòs, qui fait partie du même terçon. Encore une hypothèse plausible mais, si possible, à vérifier.

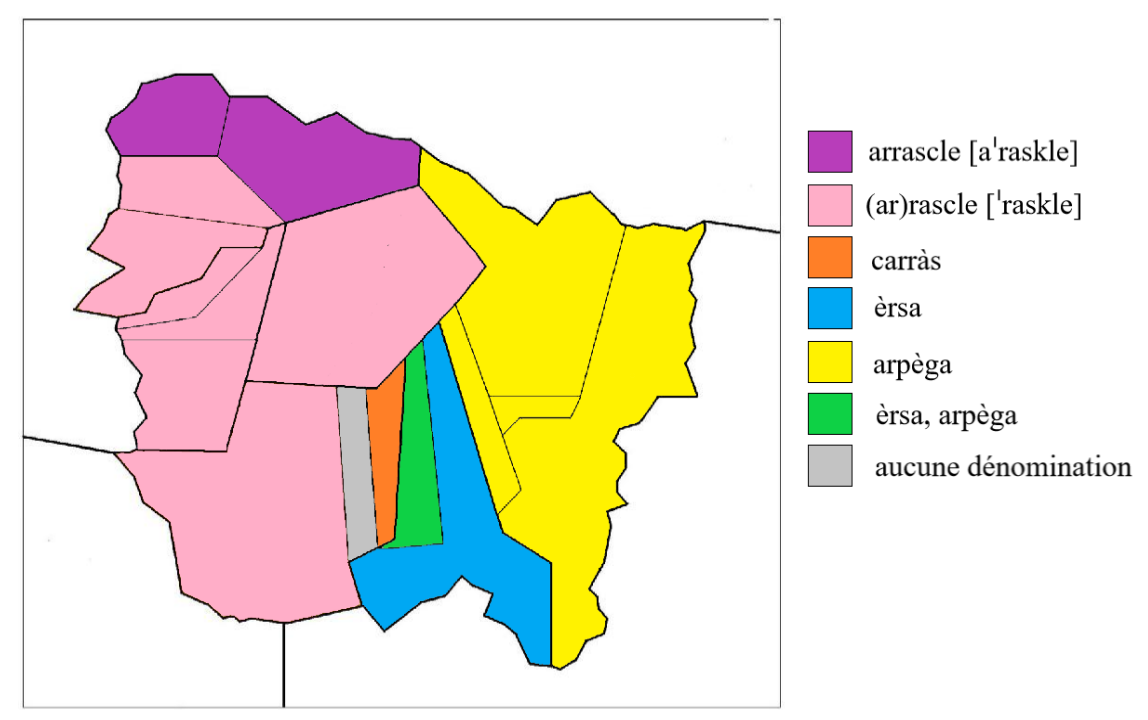

Carte 3. Herse

\section{Conclusions}

a) Même si Coromines le situe un peu partout dans le Val d'Aran (mais non pas à Les ou à Bausen) avec des applications et des définitions diverses, la vérité est que - du moins de nos jours - (ar)rastèth est surtout le mot qui désigne un râteau quelconque dans le terçon des Quate Lòcs (Canejan, Bausen, Les et Bossòst), dont le comportement linguistique ne fait pas exception à ce que l'on retrouve dans l'ensemble du domaine occitan, où des dérivés de RASTELLU existent (presque) partout. D’après nos données, en dehors de cette zone nord-occidentale, ce terme n'est pas tellement utilisé en Val d'Aran, au point que la plupart de nos informateurs le refusent ou l'attribuent à d'autres villages que les leurs. Toutefois, des survivances locales de (ar)rastèth désignant des outils similaires au râteau - telles que celle d'Aubèrt - doivent exister (et probablement elles 
étaient plus nombreuses par le passé), notamment pour nommer des crocs à petit nombre de pointes, mais non pas l'outil muni de nombreuses dents.

b) Le terme utilisé dès Arres et Vilamòs jusqu’à Escunhau pour désigner le râteau (pourvu, en général, d'un certain nombre de dents) est pletera (<*APPLIC(I)TORIA), un mot isolé en aranais qui couvre la zone centrale de la vallée (terçons de Marcatosa et de Castièro) et une partie du Baish Aran (terçon de Lairissa). Coromines a bien décelé ce fait et proposé une étymologie convenable pour ce mot.

c) Le râteau est nommé (ar)rascle partout en Naut Aran, à partir de Garòs et jusqu'à Bagergue et Tredòs. Cette appellation peut être mise en relation avec le fait que le catalan se sert parfois de rascle pour désigner le râteau, mais n'oublions pas que cette langue utilise surtout ce mot pour nommer la herse (même à des endroits très proches du Val d'Aran). Par ailleurs, cette polysémie catalane peut avoir été un facteur déterminant dans l'attribution, de la part de Coromines, de (ar)rascle 'râteau' à Bausen, où ce mot désigne en réalité la herse.

d) Les données de Coromines sur les désignations de la herse en aranais sont (presque) inexistantes. En tant que 'herse', (ar)rascle s'étend jusqu'aux portes du Naut Aran. II n'y a qu'un village - Betren - où nous n'ayons pas pu obtenir de dénomination pour cet outil. En même temps, il n'y a - à notre connaissance - qu'un seul point central faisant exception à la prépondérance de (ar)rascle 'herse'. II s'agit d'Escunhau, où nos enquêtes ont relevé le terme carràs. Ce mot, bien connu en occitan, est à mettre en relation avec les dénominations d'assemblages de pièces de bois - éventuellement roulés sur le sol - qu'on retrouve dans certaines zones du domaine occitan (tels que les traîneaux de certains villages ariégeois), mais surtout avec ce qu'on a pu constater dans des pays tels que le Rouergue, où carràs peut aussi désigner la herse après avoir subi le même glissement sémantique qu'à certains endroits du Val d’Aran.

e) En haut-aranais, alors que le nom du râteau est (ar)rascle (qui, rappelons-le, désigne la herse dans la plupart des localités situées dans le centre et dans la partie basse 
de la vallée), la herse est appelée soit èrsa, soit arpèga. Ces mots, à peine attestés jusqu'ici, méritent des précisions quant à leur répartition géographique et leur origine. Arpèga est le seul terme utilisé à Pujòlo. Èrsa, de son côté, est employé dans le terçon d'Arties e Garòs, même si à Garòs on connaît aussi arpèga (ce qui suggère un enracinement plus profond, voire plus précoce, de èrsa à Arties). Quoique éloigné de la frontière, il y a des raisons pour croire que èrsa est un francisme détaché de l'aire garonnaise-toulousaine où ce mot est prépondérant. Arpèga est à mettre en relation avec son homologue landais (également arpèga), mais aussi avec les déverbaux de HIRPICARE qui se trouvent de l'autre côté de la frontière politique, notamment en Ariège.

f) En combinant les dénominations désignant le râteau et la herse en Val d'Aran, on compte jusqu'à neuf possibilités, que nous allons essayer d'illustrer à l'aide d'une table.

\begin{tabular}{|c|c|c|c|}
\hline Terçon & Village & Râteau & Herse \\
\hline \multirow[t]{2}{*}{ Es Quate Lòcs } & Bausen, Canejan (1) & arrastèth [aras'tet/] & arrascle [a'raskle] \\
\hline & Les, Bossòst (2) & (ar)rastèth [ras'tet] & (ar)rascle ['raskle] \\
\hline Lairissa & Arres, Vilamòs, Es Bòrdes (3) & pletera [ple'tera] & (ar)rascle ['raskle] \\
\hline Marcatosa & Aubèrt (4) & $\begin{array}{c}\text { pletera [ple'tera] } \\
\text { ((ar)rastèth 'croc à } \\
\text { fumier') }\end{array}$ & (ar)rascle ['raskle] \\
\hline \multirow[t]{3}{*}{ Castièro } & Vielha (3) & pletera [ple'tera] & (ar)rascle ['raskle] \\
\hline & Betren (5) & pletera [ple'tera] & - \\
\hline & Escunhau (6) & pletera [ple'tera] & carràs [ka'ras] \\
\hline \multirow[t]{2}{*}{ Arties e Garòs } & Garòs (7) & (ar)rascle ['raskle] & $\begin{array}{c}\text { èrsa ['Ersa] } \\
\text { arpèga [ar'peya] }\end{array}$ \\
\hline & Arties (8) & (ar)rascle ['raskle] & èrsa ['Ersa] \\
\hline Pujòlo & $\begin{array}{c}\text { Gessa, Salardú, Tredòs, } \\
\text { Bagergue (9) }\end{array}$ & (ar)rascle ['raskle] & arpèga [ar'peya] \\
\hline
\end{tabular}

Table 1. Désignations du râteau et de la herse en Val d'Aran 
g) En résumé. Pour désigner le râteau, il y a trois formes possibles : (ar)rastèth (terçon des Quate Lòcs), pletera (terçons de Lairissa, de Marcatosa et de Castièro) et (ar)rascle (terçons d'Arties e Garòs et de Pujòlo). Pour nommer la herse, on se sert de (ar)rascle dans la zone basse et la partie centrale du Val d'Aran, à l'exception d'un village isolé qui utilise carràs (Escunhau) par un glissement sémantique. Arpèga est le mot de Pujòlo, tandis qu'Arties et Garòs - même si ce dernier village connaît arpèga aussi emploient la dénomination èrsa.

\section{Références}

ADEMÁ, Casimiro (1966) Estudio sobre el dialecto aranés, Barcelona: Occitania.

ALC = GRIERA, Antoni (1962-1964) Atlas lingüistic de Catalunya, 8 vol., Barcelona: Abadia de Sant Cugat del Vallès [réimpression des volumes I-V. $1^{\mathrm{e}}$ édition 1923-1936].

ALDC = VENY, Joan \& Lídia PONS (2001-2016) Atles lingüístic del domini català, 8 vol, Barcelona: IEC.

ALF = GILLIERON, Jules \& Edmont EDMONT (1902-1910) Atlas linguistique de la France, 9 vol., Paris: Honoré Champion.

ALG = SEGUY, Jean (dir.) (1954-1973) Atlas linguistique et ethnographique de la Gascogne, 6 vol., Paris: CNRS.

ALIBERT, Louis (1997) Dictionnaire occitan-français selon les parlers languedociens, Toulouse: IEO [5 $5^{\mathrm{e}}$ édition; $1^{\mathrm{e}}$ édition 1966$]$.

ALVA = GRIERA, Antoni (1973) Atlas lingüístic de la Vall d'Aran, Barcelona: Instituto internacional de cultura románica.

BALLARIN CORNEL, Ángel (1978) Diccionario del benasqués, Zaragoza: Talleres gráficos "La Editorial" [2 $2^{\mathrm{e}}$ édition; $1^{\mathrm{e}}$ édition 1971].

BARÈs, Verònica (2001) Étude sur la variation linguistique du gascon du Val d'Aran à partir d'un corpus de textes oraux, Toulouse: Université de Toulouse - Le Mirail [mémoire de DEA, inédit].

BARNILS, Pere (1914) “Notes sobre l'aranès”, Butlletí de dialectologia catalana, I, 48-56.

$\mathrm{BEC}$, Pierre (1968) Les Interférences linguistiques entre gascon et languedocien dans les parlers du Comminges et du Couserans, Paris: PUF. 
BENDEL, Hugo (1931) Beiträge zur Kenntnis der Mundart von Lescun (Bass.-Pyr.), Tübingen: Biberacher Verlagsdruckerei.

BESSON, Madeleine \& Françoise BESSON (2005) Sur les chemins du Val d'Aran. Ses habitants, ses mots, ses fleurs, Nîmes: Lacour.

BLOCH, Oscar \& Walther von WARTBURG (1975) Dictionnaire étymologique de la langue française, Paris: PUF [6 $6^{\mathrm{e}}$ édition; $1^{\mathrm{e}}$ édition 1932 ]

BoIsGONTIER, Jacques (2001) Félix Arnaudin. Dictionnaire de la Grande-Lande, vol. I, Bordeaux: Parc naturel régional des Landes de Gascogne/Éditions Confluences.

CAMPA, María del Carmen (1983) “El aranés. Dialecto gascón en tierra española. Estudio de dialectología. Fonética histórica", in M. del C. Campá et alii, Ûn libret dera Val d’Aran, Barcelona: Vinyes, 30-52.

CARRERA, Aitor (2004) "Bilanç scientific d'ua epòca istorica: es dialectològs catalans e er occitan dera Val d'Aran", Lingüistica occitana, 2, 1-21 [en ligne: http://linguistica-oc.com/wpcontent/uploads/2013/06/Linguistica-Occitana-2-Carrera.pdf]

CARRERA, Aitor (2006) "L'aportació de Joan Coromines a l'occità de la Vall d'Aran", in A. M. Badia i Margarit (éd.), Homenatge de l'IEC a Joan Coromines en el centenari de la seva naixença, Barcelona: IEC, 81-105.

CARRERA, Aitor (2007) Gramatica aranesa, Lérida: Pagès.

CARRERA, Aitor (2008) Entre dues frontères. Estudis de lingüistica occitana, Lérida: Pagès.

CARRERA, Aitor (2014) "Una qüestió fonètica entre el gascó i el llenguadocià. La pròtesi de [a] davant de R- en l'occità de la Vall d'Aran i de l'Alt Comenge immediat", in C. Passet (dir.), Actes du 14e Colloque des langues dialectales, Monaco: EGC, 155-177.

CARRERA, Aitor (2015) "L'occità de la Vall d'Aran davant dels parlars gascons veïns. Implicacions lingüístiques de la separació política", in Institut d’Estudis Catalans - Secció HistòricoArqueològica, La Reintegració de la Vall d'Aran a Catalunya, Barcelone: IEC, 63-96.

CARRERA, Aitor (2017) "De cap a un nau atlàs lingüistic dera Val d'Aran", in A. Carrera/I. Grifoll (éds.), Occitània en Catalonha : de tempses novèls, de novèlas perspectivas. Actes de I'Xlen Congrès de l'Associacion internacionala d'estudis occitans, Barcelone/Lérida: Generalitat de Catalunya/Diputació de Lleida, 103-124.

CARRERA, Aitor (sous presse) "Notes de toponymie gasconne. À propos de quelques noms de lieux de Bossòst (Val d'Aran)", in Actes du XVIII Colloque de la Société française d'onomastique (Toulouse, 2018). 
Cenac-Moncaut [Justin] ([1863] 1993) Dictionnaire gersois-français français-gersois, Nîmes: Lacour [réédition de l'ouvrage original paru en 1863, Dictionnaire gascon-français. Dialecte du département du Gers].

ConDO, J. [Jusèp] (1914) “Vocabulari aranès”, Butlletí de dialectologia catalana, III, 1-27.

COROMINAS, Juan [COROMINES, Joan] (1931) Vocabulario aranés, Barcelona: Imprenta de la Casa de Caridad.

COROMINES, Joan (1990) El Parlar de la Vall d'Aran: gramàtica, diccionari i estudis lexicals sobre el gascó, Barcelona: Curial.

CREMONA, Joseph (1956) The Dialect of the Vallée d'Aure, Londres: Université de Londres [thèse inédite].

DBGM = PALAY, Simin (1980) Dictionnaire du béarnais et $d u$ gascon modernes, Paris: CNRS [3 ${ }^{\mathrm{e}}$ édition; $1^{\mathrm{e}}$ édition 1932-1934].

DCVB = ALCOVER, Antoni M. \& Francesc de B. Moll (1985) Diccionari català-valencià-balear, 10 vol., Palma de Mallorca: Moll [réimpression; 1932-1962].

DECat = COROMINES, Joan (1980-2001) Diccionari etimològic $i$ complementari de la llengua catalana, 10 vol., Barcelona: Curial/La Caixa.

DECH = COROMINAS, Joan [Coromines, Joan] \& José A. PASCUAL (1980-1991) Diccionario crítico etimológico castellano e hispánico, 6 vol., Madrid: Gredos.

DUPLEICH (1843) Dictionnaire patois-français ou Choix intéressant de mots patois à l'usage de I'arrondissement de Saint-Gaudens, Saint-Gaudens: J.-M. Tajan.

FAHRHOLZ, Günther (1931) Wohnen und Wirtschaft im Bergland der oberen Ariège. Sach- und Wortkundliches aus den Pyrenäen, Hamburg: Seminar für romanische Sprachen und Kultur.

FERRAZ y CASTAN, Vicente (1934) Vocabulario del dialecto que se habla en la Alta Ribagorza, Madrid: Tipografía de Archivos.

FEW = WARTBURG, Walther von (1922-...) Französisches etymologisches Wörterbuch, Bonn/Bâle: F. Klopp/Helbing \& Lichtenhahn/Zbinden.

FOSSAT, Jean-Louis (1971) La Formation du vocabulaire gascon de la boucherie et de la charcuterie, Toulouse: Imprimerie Menard.

GINESTET, Joëlle (2011) Los Mòts de Jan-Guiraud Dastròs, poèta gascon deu començament deu sègle XVII, Orthez: Los Quasèrns de Lomanha.

GIPP = RONJAT, Jules ([1930-1941] 1980) Grammaire istorique [sic] des parlers provençaux modernes, 4 vol., Genève/Marseille: Slatkine/Laffitte [réédition de l'ouvrage paru en 19301941]. 
GUILHEMJOAN, Patric (dir.) (2003-2005) Dictionnaire français-occitan (gascon), 2 vol., Orthez: Per Noste.

HEYNS, Karl (1938) Wohnkultur, Alp- und Forstwirtschaft im Hochtal der Garonne, Hambourg: Seminar für romanische Sprachen und Kultur.

KRÜGER, Fritz ([1939] 1996) Los altos Pirineos. Vol. III. Las labores del camp (segunda parte). Economía agraria, Tremp/Huesca/Zaragoza: Garsineu/Diputación de Huesca/Diputación general de Aragón [traduction de l'ouvrage allemand paru en 1939].

KRÜGER, Fritz ([1945] 1997) Los altos Pirineos. Vol. IV. Manufacturas caseras, indumentaria, industrias, Tremp/Huesca/ Zaragoza: Garsineu/Diputación de Huesca/Diputación general de Aragón [traduction de l'ouvrage allemand paru en 1945].

LAGARDE, André (1991) Le Trésor des mots d'un village occitan. Dictionnaire du parler de Rivel (Aude), Toulouse: IEO.

LAURENT, Jean-Pierre (2002a) Les Dialectes du Séronais. La Bastide-de-Sérou, Castelnau-Durban, le Mas-d'Azil. Grammaire et dictionnaire, s.l.: chez l'auteur.

LAURENT, Jean-Pierre (2002b) Le Dialecte gascon d'Aulus. Grammaire et dictionnaire, s.l.: chez l'auteur.

LE NAIL, Jean-François \& EyGUN, Jean (2015) François Marsan et Jean Soulé-Venture. Lexiques gascons inédits, Pau: Camins.

LESPY, Vastin \& Paul RAYMOND ([1887] 1998) Dictionnaire du béarnais ancien et moderne, Pau: Marrimpouey Jeune [réédition de l'ouvrage original paru en 1887].

LUCHAIRE, Achille (1881) Recueil de textes de l'ancien dialecte gascon, Paris: Maisonneuve.

MOUREAU, Pierre ([1870] 1997) Diccionari gascon-français. Dictionnaire français-gascon, suivant les parlers maritimes, Pau: Princi Néguer [réédition de l'ouvrage original paru en 1870, Dictionnaire du patois de la Teste].

ReI BethVEDER, Nicolau (2004) Dictionnaire français-occitan. Gascon toulousain, Bouloc: IEO.

ROQUES, Gabriel ([1913] 1977) Grammaire gasconne: dialecte de l'agenais. Glossaire gasconlanguedocien, Marseille: Laffitte [réimpression de l'ouvrage original, paru en 1913].

ROHLFS, Gerhard (1977) Le Gascon. Études de philologie pyrénéenne, Tübingen/Pau: Max Niemeyer/Marrimpouey Jeune [ $3^{\mathrm{e}}$ édition; $1^{\mathrm{e}}$ édition 1935].

SCHMITT, Alfons Theo (1934) La Terminologie pastorale dans les Pyrénées Centrales, Paris: Droz.

SCHÖNTHALER, Willy (1937) Die Mundart des Bethmale-Tales (Ariège), Tübingen: Eugen Göbel.

SEGUY, Jean (1956) “Les cartes auxiliaires de l'Atlas linguistique et ethnographique de la Gascogne, Essai d'aréologie méthodique", Via Domitia, III, 35-62. 
Dialectologia 25 (2020), 87-113.

ISSN: 2013-2247

SÉGUY, Jean (1973) "La fonction minimale du dialecte", in G. Straka/P. Gardette (éds.), Les Dialectes romans de France à la lumière des atlas régionaux, Paris: CNRS, 27-42.

Soler I SANTALO, Juli ([1906] 1998) La Vall d'Aran, Tremp: Garsineu [réédition de l'ouvrage original paru en 1906].

THESOC = Thesaurus occitan. Base de données linguistiques [en ligne: http://thesaurus.unice.fr/index.html]

TDF = MISTRAL, Frédéric ([1887] 1979) Lou Trésor dóu Felibrige, 2 vol., Raphèle-lès-Arles: Marcel Petit [réédition de l'ouvrage paru en 1887].

VAYSSIER, Aimé (1879) Dictionnaire patois-français du département de l'Aveyron, Rodez: E. Carrère.

VERGÉS, Frederic (2009) Petit diccionari castelhan-aranés (occitan)-catalan-francés aranés (occitan)-castelhan-catalan-francés, Vielha: Conselh generau d'Aran $\left[3^{\mathrm{e}}\right.$ édition; $1^{\mathrm{e}}$ édition 1991]. 